\title{
FREQUÊNCIA DE DISTRIBUIÇÃO DE EVAPOTRANSPIRAÇÃO DA BACIA HIDROGRÁFICA DO A̧LTO JURUÁ, OESTE AMAZÔNICO
}

\author{
EVAPOTRANSPIRATION DISTRIBUTION FREQUENCY OF THE ALTO JURUÁ \\ HYDROGRAPHIC BASIN, WEST AMAZON
}

\author{
Jefferson Vieira José 1* $^{*}$, Lívia Braz Pereira ${ }^{2}$, Moisés Damasceno Souza ${ }^{\mathbb{D}}$, Kelly Nascimento \\ Leite $^{4}{ }^{\oplus}$, Hugo Motta Ferreira Leite ${ }^{5}{ }^{\oplus}$, Lucas da Costa Santos ${ }^{6}{ }^{\oplus}$ \\ ${ }^{1}$ Prof. Doutor em Engenharia de Sistemas Agrícolas, Universidade Federal do Acre, Cruzeiro do Sul, AC, Brasil. \\ ${ }^{2}$ Graduanda em Agronomia, Universidade Federal do Acre, Cruzeiro do Sul, AC, Brasil. \\ ${ }^{3}$ Graduando em Agronomia, Universidade Federal do Acre, Cruzeiro do Sul, AC, Brasil. \\ ${ }^{4}$ Profa. Doutora em Engenharia Agrária, Universidade Federal do Acre, Cruzeiro do Sul, AC, Brasil. \\ ${ }^{5}$ Prof. Doutor em Agronomia, Universidade Federal do Acre, Cruzeiro do Sul, AC, Brasil. \\ ${ }^{6}$ Prof. Doutor em Engenharia de Sistemas Agrícolas, Universidade Federal dos Vales do Jequitinhonha e Mucuri, Teófilo Otoni, MG, Brasil.
}

RESUMO: A evapotranspiração é uma importante variável para a prática sustentável da agricultura irrigada, é imprescindível que haja estudos que visem uma melhor compreensão e aplicabilidade desta variável em diferentes regiões. $\mathrm{O}$ trabalho tem por objetivo analisar a frequência de distribuição da evapotranspiração de referência, estimada pelo método de Penman-Monteith-FAO, em períodos acumulados de 1, 5, 10, 20 e 30 dias, para os níveis de probabilidade 50, 70, 80, 90 e 95\%, para a Bacia Hidrográfica do Alto Juruá. Dados de 33 anos de evapotranspiração foram ajustados para seis modelos de distribuição de probabilidade (Lognormal, Weibull, Gama, Normal, Logística e Cauchy). Observamos que, de todas as distribuições testadas, apenas a de Cauchy não foi eficaz na modelagem dos dados. Ademais, ao adotar como parâmetros o período acumulado de 20 dias e a probabilidade de $80 \%$, verificamos subdimensionamento em sistemas de irrigação por parte do valor médio. Através dos parâmetros escolhidos, obteve-se ETo corresponde a $6,34 \mathrm{~mm} \mathrm{dia}^{-1}$, sendo este o valor indicado para dimensionar os sistemas de irrigação na região.

Palavras-chave: Irrigação, Penman-Monteith, probabilidade

\begin{abstract}
Evapotranspiration is an important variable for the sustainable practice of irrigated agriculture, there must be studies that aim at a better understanding and applicability of this variable in different regions. The job aims to analyze the frequency of distribution of reference evapotranspiration, estimated by the Penman-Monteith-FAO method, in cumulative periods of $1,5,10,20$, and 30 days, for the 50, 70, 80 probability levels, 90 and 95\%, for the Alto Juruá Hydrographic Basin. The data of 33 years of evapotranspiration were adjusted for six probability distribution models (Log-normal, Weibull, Gamma, Normal, Logistics, and Cauchy). We observed that, of all the tested distributions, only Cauchy's was not effective in modeling the data. We observed that, of all the tested distributions, only Cauchy's was not effective in modeling the data. Also, by adopting as parameters the accumulated period of 20 days and the probability of $80 \%$, we verified under-dimensioning in irrigation systems by the average value. Through the chosen parameters, ETo corresponds to $6.34 \mathrm{~mm} \mathrm{day}^{-1}$, which is the value indicated for dimensioning the irrigation systems in the region.
\end{abstract}

Key words: Irrigation, Penman-Monteith, probability 


\section{INTRODUÇÃ̃O}

O Estado do Acre possui um potencial irrigável de 700 mil de hectares, sendo utilizado apenas $0,3 \%$, comparado a área irrigada atual de 1826 hectares (ANA, 2017). Dada a perspectiva do aumento de áreas irrigada no Acre, novas estratégias de uso da água devem ser implementadas na Bacia do Alto Juruá. Concomitantemente, deve-se buscar formas de aumentar a eficiência dos sistemas de irrigação, permitindo uma redução na pressão pelo uso da água na bacia hidrográfica (CÓRCOLES et al., 2010; IBGE, 1998; IBGE, 2017).

Uma das principais dificuldades para a implantação de projetos de irrigação é definir, criteriosamente, o valor da evapotranspiração de referência (ETo) que servirá de base para o dimensionamento dos sistemas, de acordo com as necessidades hídricas das culturas (BACK, 2007; SANTOS et al., 2014). A grande variabilidade de valores assumidos pelos elementos meteorológicos durante o período de máxima exigência hídrica das culturas, acarreta considerável dispersão dos valores da ETo (JOSÉ et al., 2019; FERNANDES et al., 2019), sugerindo uma análise da distribuição de frequência dos valores estimados, para fins de dimensionamento de projetos (SAAD; SCALOPPI, 2002; DENSKI; BACK, 2015).

A ETo provável é a taxa de ETo estimada a partir de uma distribuição de probabilidade, que pode ser igualada ou superada a determinado nível de probabilidade, definido com base no histórico da ETo e selecionados com base em critérios econômicos (FIETZ et al., 1997; ALBUQUERQUE; DURÃES, 2008; DENSKI; BACK, 2015). Nas regiões úmidas são sugeridos valores de 80 a $90 \%$ da ETo máxima (BERNARDO, SOARES;
MANTOVANI, 2009). A duração do período crítico das culturas anuais é sugerida de 10 a 30 dias que servirá de base para o dimensionamento do sistema (SAAD; SCALOPPI, 1988).

Diante do exposto, este trabalho teve como objetivo avaliar o ajuste de seis distribuições de probabilidade aos dados de ETo, considerando um período de 33 anos, calculados a ETo acumulada em intervalos de $1,5,10,20$ e 30 dias com diferentes níveis de probabilidade.

\section{MATERIAL E MÉTODOS}

A evapotranspiração de referência (ETo) foi estimada utilizando dados climáticos de uma série histórica de 33 anos (1980 a 2013) obtida em três estações meteorológicas automáticas, localizadas na Bacia Hidrográfica do Alto Juruá (BHAJ) no Estado do Acre, sendo elas: Cruzeiro do Sul, Porto Walter e Marechal Thaumaturgo. A BHAJ está localizada entre as longitudes de 70 e $74^{\circ} \mathrm{W}$ e as latitudes de 7 e $10^{\circ} \mathrm{S}$ (Figura 1), possui tipologia climática tipo Af (Köppen-Geiger), caracterizada por clima tropical equatorial, sem estação seca (ALVARES et al., 2013).

Utilizou-se o método de PenmanMonteith-FAO (ALLEN et al., 1998) para estimativa da ETo, requerendo dados meteorológicos de temperatura do ar (máxima, mínima e média), umidade relativa do ar (média), radiação solar, velocidade do vento e pressão atmosférica, que foram obtidos a partir da base de dados meteorológicos desenvolvido por Xavier et al. (2015), a partir de técnicas de interpolação, por meio do software REF-ET. Os valores de ETo foram acumulados em períodos consecutivos de 1 (ETo1), 5 (ETo5), 10 (ETo10), 20 (ETo20) e 30 (ETo30) dias. 




Figura 1. Localização das estações pluviométricas na Bacia Hidrográfica do Alto Juruá

A análise de distribuição de frequência das estimativas da evapotranspiração máxima de referência acumulada $\left(\mathrm{ETo}_{\mathrm{ac}}\right)$ foram ajustadas para seis distribuições de probabilidade, como segue: (i) Log-Normal, (ii) Weibull, (iii) Gama, (iv) Normal, (v) Logística e (vi) Cauchy (NAGHETTINE; PINTO, 2007). A ETo ac para os níveis de probabilidade de 50, 70, 80, 90 e 95\%, corresponde ao tempo de retorno (T) de 2, 3, 5, 10 e 20 anos, respectivamente. Os parâmetros das distribuições de probabilidade foram estimados utilizando-se o método da máxima verossimilhança. A escolha da distribuição de probabilidade que melhor se ajustou aos dados foi mediante o menor valor do Critério de Informação de Akaike (AIC). Para verificar o ajuste dos dados de ETo às distribuições de probabilidade aplicou-se o teste de Anderson-Darling $\quad(\mathrm{P}<0,05)$ (NAGHETTINE; PINTO, 2007).

Foram estimados a irrigação diária total necessária e o volume bruto de irrigação por planta por dia provável, no período de máxima demanda, considerando dois projetos de irrigação para as culturas do café e tomate. Utilizou os parâmetros do projeto de irrigação por gotejamento com faixa molhada continua com porcentagem de área coberta pela cultura (Fc) de 0,56-0,78; coeficiente da cultura máximo (Kc) de 1,10-1,15; uniformidade de emissão (EU) de 0,90-0,80; intervalo entre irrigações (TR) de 5-1 dias e espaçamento 0,60,5 m entre si, e 3,05-0,75 $\mathrm{m}$ e entre linhas, para a projeto do café e tomate, respectivamente (FRIZZONE et al., 2012).

Foi utilizado o software livre $\mathrm{R}$ Statistical ${ }^{\circledR}$ (R CORE TEAM, 2020) e os seus pacotes para obtenção e análise dos dados. O banco de dados de Xavier et al. (2015) foram extraídos com os pacotes raster (HIJMANS, 2020) e ncdf4 (PIERCE, 2019). A manipulação dos dados de $E T_{a c}$ e o ajuste de modelos de distribuição foi realizado por meio do pacote fitdistrplus (DELIGNETTEMULLER; DUTANG, 2015) e, do teste de aderência, com o pacote ADGofTests (BELLOSTA, 2011). Gráficos foram realizados com pacotes ggplot2 (WICKHAM, 2016) e gridExtra (AUGUIE, 2017). 


\section{RESULTADOS E DISCUSSÃO}

A partir dos dados obtidos observa-se que, com o aumento dos dias acumulados, os valores médios da ETo diminuem (Figura 2). Esta tendência também foi observada por Fietz et al. (1997) ao analisar a frequência de evapotranspiração para a região de DouradosMS, por Saad et al. (2002) na região de Piracicaba-SP e por Back (2007) na região de Urussanga - SC. Segundo Saad e Scaloppi (1988) esta tendência normalmente ocorre em análises de frequência da evapotranspiração.
Os valores médios de $\mathrm{ETo}_{\mathrm{ac}}$ entre as estações foram semelhantes para cada período acumulado. $\mathrm{Na}$ ETo com 10 dias acumulados a média entre as três estações foi de $6,75 \pm 0,25 \mathrm{~mm} \mathrm{dia}{ }^{-1}$, com valores de $6,76 \pm 0,26 ; 6,75 \pm 0,27$ e $6,75 \pm 0,27$ $\mathrm{mm}$ dia $^{-1}$ nas estações Cruzeiro do Sul, Marechal Thaumaturgo e Porto Walter, respectivamente (Figura 2B). Ademais, a própria variação média observada nos gráficos, bem como o desvio padrão, demostra que há um aumento da ETo com o tempo (Figura 2A).
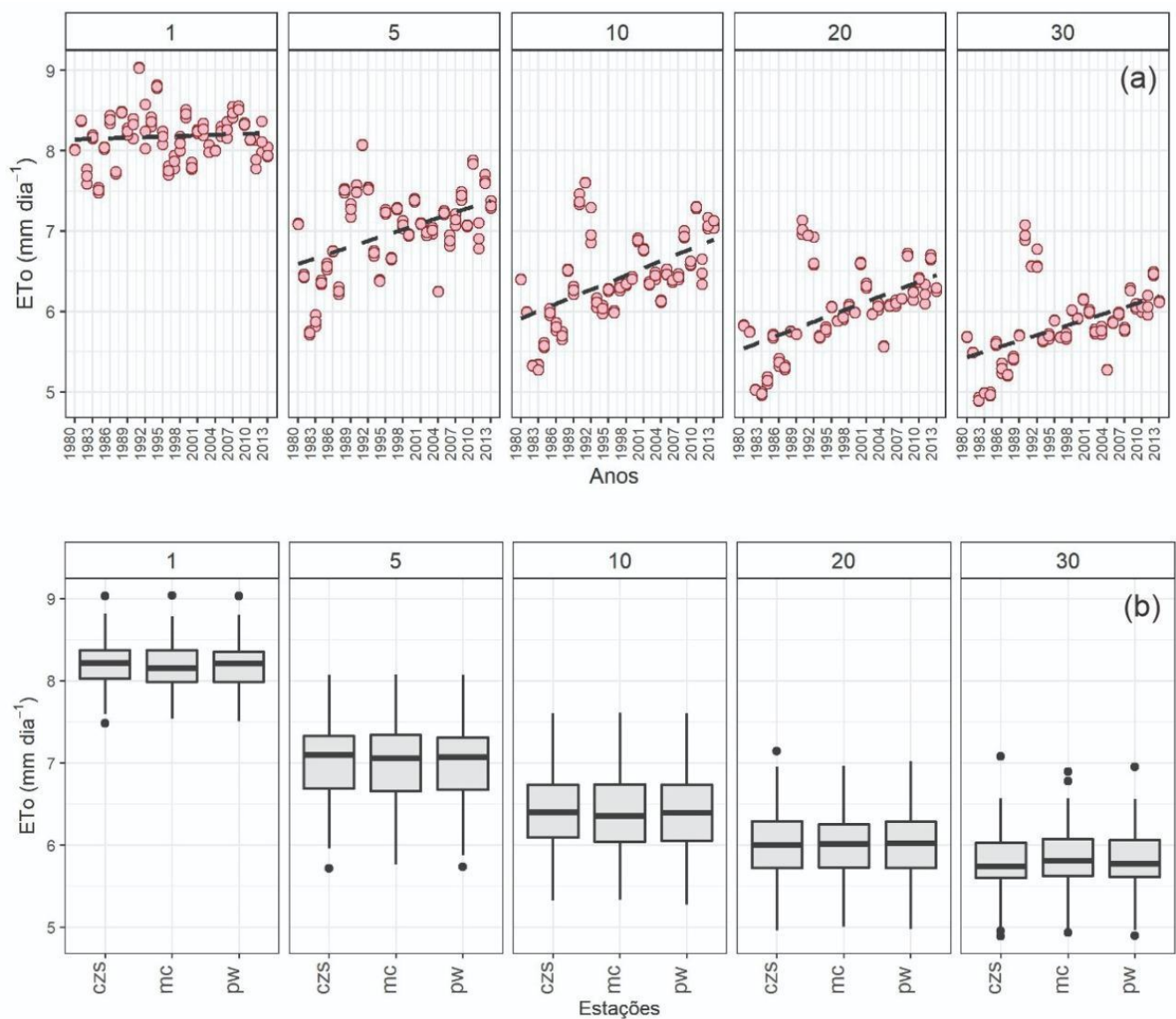

Figura 2. Variação média de evapotranspiração de referência provável na escala diária e em períodos acumulados de 1, 5, 10, 20 e 30 dias, para o período de 1980 a 2013 e entre as estações climáticas de Cruzeiro do Sul (czs), Porto Walter (pw) e Marechal Thaumaturgo (mc) na Bacia Hidrográfica do Alto Juruá, Acre

Na Tabela 1 verifica-se que todos os modelos estavam ajustados aos dados de ETo, e posteriormente, que as distribuições estavam estatisticamente adequadas a representar os dados de evapotranspiração de referência para a Bacia Hidrográfica do Alto Juruá para seus respectivos dias acumulados. 
Frequência de distribuição de evapotranspiração da bacia hidrográfica do Alto Juruá, oeste amazônico

Tabela 1. Avaliação das distribuições de probabilidade pelo Critério de Informação de Akaike (AIC)

\begin{tabular}{|c|c|c|c|c|c|c|}
\hline \multirow{2}{*}{$\begin{array}{c}\text { Período } \\
\text { Acumulado } \\
\text { de ETo (dias) }\end{array}$} & Log-Normal & Weibull & Gama & Cauchy & Normal & Logística \\
\hline & \multicolumn{6}{|c|}{ Cruzeiro do Sul } \\
\hline 1 & 22,40 & 28,20 & 22,38 & 31,46 & 22,40 & $21,88^{*}$ \\
\hline 5 & 168,35 & $166,26^{*}$ & 167,85 & 177,16 & 176,03 & 167,72 \\
\hline 10 & 215,20 & 218,91 & $215,14^{*}$ & 224,03 & 215,26 & 215,74 \\
\hline 20 & 258,69 & 261,20 & 258,50 & 267,74 & $258,37 *$ & 258,78 \\
\hline \multirow[t]{2}{*}{30} & $280,87 *$ & 286,49 & 280,89 & 289,02 & 281,17 & 280,90 \\
\hline & \multicolumn{6}{|c|}{ Marechal Thaumaturgo } \\
\hline 1 & $22,05^{*}$ & 30,92 & 22,19 & 22,54 & 22,55 & 22,55 \\
\hline 5 & 167,06 & 165,94 & 166,61 & 176,60 & $165,89 *$ & 166,40 \\
\hline 10 & 216,28 & 220,09 & $216,27 *$ & 225,43 & 216,46 & 217,20 \\
\hline 20 & 257,71 & 260,44 & 257,59 & 267,03 & $257,57 *$ & 258,28 \\
\hline \multirow[t]{2}{*}{30} & 278,86 & 283,46 & $278,84 *$ & 286,71 & 279,00 & 278,97 \\
\hline & \multicolumn{6}{|c|}{ Porto Walter } \\
\hline 1 & 20,36 & 28,57 & 20,45 & 29,80 & 20,67 & $20,19 *$ \\
\hline 5 & 167,03 & $165,18^{*}$ & 166,53 & 175,72 & 165,72 & 166,20 \\
\hline 10 & 215,60 & 218,80 & $215,49 *$ & 224,12 & 215,51 & 216,03 \\
\hline 20 & 257,09 & 258,91 & 256,88 & 266,94 & $256,67 *$ & 257,35 \\
\hline 30 & 278,69 & 282,86 & $278,61^{*}$ & 287,03 & 278,67 & 278,67 \\
\hline
\end{tabular}

*melhor distribuição de probabilidade pelo Critério de Informação de Akaike (AIC)

Os melhores modelos de distribuição de probabilidade aptos a representar os dados de ETo durante seus respectivos dias acumulados estão dispostos na Tabela 2, bem como os seus parâmetros e o valor do teste de AndersonDarling para cada distribuição. A distribuição Gama é a que mais aparece, um total de cinco vezes, onde se mostra presente para os períodos acumulados de 10 e 30 dias sendo adequada para os dados das estações de Cruzeiro do Sul, Porto Walter e Marechal Thaumaturgo durante o acumulado de 10 dias, enquanto para um período acumulado de 30 dias, a distribuição de probabilidade se adequa apenas as estações de Porto Walter e Marechal Thaumaturgo. Percebe-se que a distribuição não é ideal para períodos acumulados menores. Essa adequação também foi observada por Silva et al. (2014) em Crateús, Quixeramobim e Tauá no Estado do Ceará ao ao analisarem dados mensais de ETo.

Tabela 2. Valores dos parâmetros e teste de Anderson-Darling dos modelos de distribuição mais adequados na determinação da probabilidade de ocorrência da evapotranspiração de referência acumulada máxima anual na Bacia do Alto Juruá

\begin{tabular}{|c|c|c|c|c|c|c|c|c|c|}
\hline \multirow{2}{*}{$\begin{array}{l}\text { Período } \\
\text { acumulado } \\
\text { (dias) }\end{array}$} & \multirow{2}{*}{$\begin{array}{l}\text { Teste de } \\
\text { Anderson- } \\
\text { Darling } \\
\text { p-valor }{ }^{\mathrm{a}}\end{array}$} & \multicolumn{2}{|c|}{$\begin{array}{l}\text { Cruzeiro do } \\
\text { Sul }\end{array}$} & \multirow{2}{*}{$\begin{array}{l}\text { Teste de } \\
\text { Anderson- } \\
\text { Darling } \\
\text { p-valor }^{\mathrm{a}}\end{array}$} & \multicolumn{2}{|c|}{$\begin{array}{c}\text { Marechal } \\
\text { Thaumaturgo }\end{array}$} & \multirow{2}{*}{$\begin{array}{l}\text { Teste de } \\
\text { Anderson- } \\
\text { Darling } \\
\text { p-valor }^{\mathrm{a}}\end{array}$} & \multicolumn{2}{|c|}{ Porto Walter } \\
\hline & & Par1 & Par2 & & Par1 & Par2 & & Par1 & Par2 \\
\hline \multirow{2}{*}{1} & \multirow{2}{*}{0,99} & $\log$ & ica & \multirow{2}{*}{0,96} & \multicolumn{2}{|c|}{ Log-Normal } & \multirow{2}{*}{0,99} & \multicolumn{2}{|c|}{ Logística } \\
\hline & & 8,2 & 0,17 & & 2,09 & 0,04 & & 8,17 & 0,17 \\
\hline \multirow{2}{*}{5} & \multirow{2}{*}{0,99} & $\mathrm{We}$ & & \multirow{2}{*}{0,94} & \multicolumn{2}{|c|}{ Normal } & \multirow{2}{*}{0,99} & \multicolumn{2}{|c|}{ Weibull } \\
\hline & & 15,24 & 36,24 & & 34,85 & 2,62 & & 15,35 & 36,08 \\
\hline \multirow{2}{*}{10} & \multirow{2}{*}{0,97} & & & \multirow{2}{*}{0,86} & \multicolumn{2}{|c|}{ Gama } & \multirow{2}{*}{0,93} & \multicolumn{2}{|c|}{ Gama } \\
\hline & & 140,56 & 2,19 & & 135,45 & 2,11 & & 138,48 & 2,16 \\
\hline \multirow{2}{*}{20} & \multirow{2}{*}{0,97} & & & \multirow{2}{*}{0,92} & \multicolumn{2}{|c|}{ Normal } & \multirow{2}{*}{0,98} & \multicolumn{2}{|c|}{ Normal } \\
\hline & & 119,97 & 10,19 & & 120,08 & 10,07 & & 119,92 & 9,94 \\
\hline \multirow{2}{*}{30} & \multirow{2}{*}{0,90} & Log-I & rmal & \multirow{2}{*}{0,94} & \multicolumn{2}{|c|}{ Gama } & & \multicolumn{2}{|c|}{ Gama } \\
\hline & & 5,16 & 0,08 & & 159,99 & 0,92 & 0,91 & 160,34 & 0,92 \\
\hline
\end{tabular}

Weibull (Par1 - escala e Par2 - forma); Gama (Par1 - taxa e Par2 - forma); Normal (Par1 - média e Par2 - desvio padrão); LogNormal (Par1 - log (média) e Par2 - log (desvio padrão); ${ }^{a}$ Teste de aderência Anderson-Darling significativo $(\mathrm{p}<0,05)$

Nota-se que as distribuições LogNormal e Normal se adequam bem a maiores e menores períodos acumulados, porém apenas para algumas das três estações climáticas. Essa 
flexibilidade de aderência da distribuição Normal a maiores e menores períodos acumulados também foi observada por Santos et al. (2011) na região de Mossoró-RN, onde a distribuição Normal se adequou a períodos acumulados de 5, 10 e 30 dias. A flexibilidade de aderência da distribuição Normal a dados de maiores e menores períodos pode ser explicada devido ao fato de que a ETo tende a ajustar-se a uma distribuição Normal, segundo Pruitt et al. (1972). A distribuição Log-Normal também se mostrou adequada para a região de Crateús para um período acumulado de 30 dias, onde, para o mesmo período acumulado, as distribuições Normal, Log-Normal e Gama se ajustaram aos dados mensais de evapotranspiração de referência para as regiões de Crateús, Quixeramobim e Tauá, segundo Silva et al. (2014). A aderência das distribuições Log-Normal e Normal a períodos acumulados maiores e menores também foi observada por Fietz et al. (1997) na região de Dourados, ao analisar dados de evapotranspiração para períodos acumulados de $5,10,15,20$ e 30 dias.

Observa-se também que a distribuição de Weibull e Logística são adequadas apenas para os menores períodos acumulados de ETo. Para a região de Mossoró, segundo Santos et al. (2011), a distribuição de Weibull se adequa melhor ao período acumulado de 10 dias (decendial), enquanto para a região de estudo deste trabalho, a distribuição se adere melhor ao período de 5 dias.

As distribuições de Logística, Weibull e Log-Normal foram as que apresentaram o pior desempenho geral, aparecendo nos períodos acumulados de 1 e 5 dias, sendo a distribuição de Log-Normal a única que ocorreu em dois períodos acumulados diferentes (1 e 30 dias). A distribuição de Cauchy não ocorreu em nenhum período acumulado, não sendo assim recomendada. Santos et al. (2011) também observaram performance insatisfatória das distribuições de Weibull e Log-Normal em seu estudo.

Com o ajuste dos dados de evapotranspiração às distribuições, foram estimados para cada ano os valores de evapotranspiração de referência com probabilidades de 50, 70, 80, 90 e 95\% (Figura 3). Para um período acumulado de ETo de 20 dias e a probabilidade de ocorrência de $80 \%$ (período de retorno de 5 anos), o valor de ETo corresponde a $6,34 \mathrm{~mm} \mathrm{dia}^{-1}$. Portanto, existe $80 \%$ de probabilidade de que o valor de ETo para um período acumulado de 20 dias não ultrapasse $6,34 \mathrm{~mm} \mathrm{dia}^{-1}$ ou, ainda, para o mesmo período, em apenas um de cada cinco anos o valor de ETo será igual ou superior a $6,34 \mathrm{~mm} \mathrm{dia}^{-1}$. Fietz et al. (1997) observaram que na região de Dourados/MS, para condições semelhantes, o valor de ETo foi correspondente a $5,94 \mathrm{~mm} \mathrm{dia}{ }^{-1}$. Na região de Cruz das Almas/BA, Santos et al. (2014) verificaram que, para um período acumulado de 30 dias, os valores de ETo variaram de 2,86 a $5,41 \mathrm{~mm} \mathrm{dia}^{-1}$ com probabilidade de ocorrência de $80 \%$.

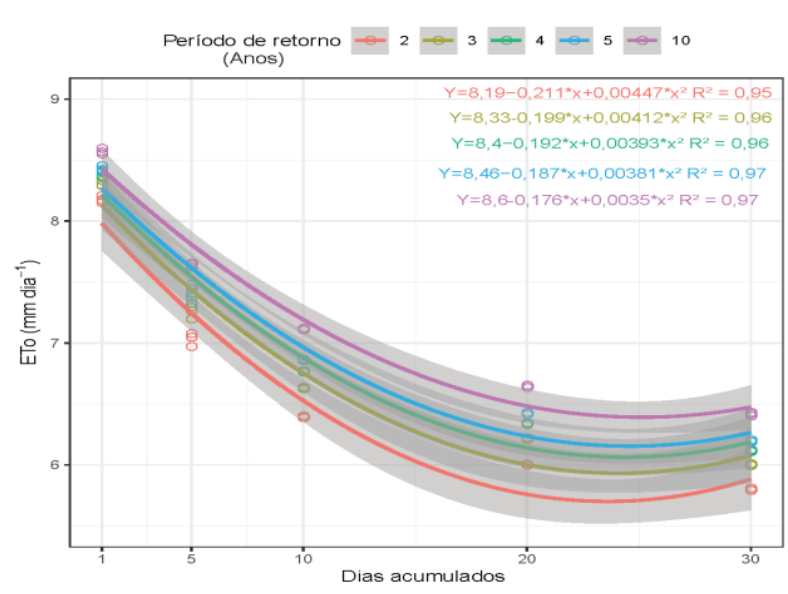

Figura 3. Variação da evapotranspiração de referência média provável de dias acumulados de $1,5,10,20$, e 30 com período de retorno de 2, 3, 5, 10 e 20 anos, na Bacia Hidrográfica do Alto Juruá

Os valores de ETo quando multiplicados por seus respectivos coeficientes de cultura, podem ser usados como parâmetros no dimensionamento de sistemas de irrigação (FIETZ et al., 1997). Segundo Saad et al. (2002), em termos de Brasil, o que se verifica, na prática, é a utilização do valor médio mensal da evapotranspiração ou a adoção do valor máximo diário do período de maior exigência hídrica da cultura irrigada.

$\mathrm{Na}$ Tabela 3 é possível comparar o valor recomendado e o valor médio mensal do mês de setembro. Ao comparar o volume bruto de irrigação (VTN) por planta por dia para a 
cultura do café, sendo o valor médio de 7,62 L planta $^{-1}$ dia $^{-1}$ e o valor recomendado de 10,59 L planta ${ }^{-1}$ dia $^{-1}$, nota-se que o valor recomendado foi superior em relação à média, havendo subdimensionamento em cerca de $28 \%$ ao se utilizar o valor médio. Saad et al. (2002) também observaram subdimensionamento por parte do valor médio usando como comparação o valor de evapotranspiração a $75 \%$ de probabilidade. Ao comparar esses mesmos valores para a cultura do tomate, também se percebe o subdimensionamento por parte do valor médio em cerca de $37 \%$, sendo o valor médio de 3,50 L planta ${ }^{-1}$ dia $^{-1}$ e o valor recomendado de 2,63 L planta ${ }^{-1}$ dia $^{-1}$.

Tabela 3. Consumo hídrico provável no período de máxima demanda para as culturas do café e tomate na Bacia do Alto Juruá, Estado do Acre.

\begin{tabular}{|c|c|c|c|c|c|c|}
\hline \multirow[t]{2}{*}{ Período de retorno } & \multirow{2}{*}{$\begin{array}{l}\text { Probabilidade } \\
\text { de ocorrência }\end{array}$} & \multirow{2}{*}{$\begin{array}{c}\mathrm{ETO}_{5} \\
\mathrm{~mm}\end{array}$} & $\begin{array}{l}\text { Parâmetros do projeto } \\
\text { de irrigação* }\end{array}$ & $\begin{array}{l}\mathrm{ETc} \\
\mathrm{mm}\end{array}$ & $\begin{array}{c}\text { ITN } \\
\mathrm{mm} \mathrm{dia}^{-1} \\
\end{array}$ & $\begin{array}{c}\text { VTN } \\
\text { L planta }{ }^{-1} \text { dia }^{-1} \\
\end{array}$ \\
\hline & & & \multicolumn{4}{|c|}{ Café - irrigação por gotejamento com faixa molhada contínua } \\
\hline 2 anos & $50 \%$ & 7,03 & \multirow{6}{*}{$\begin{array}{c}\text { Espaçamento }-0,6 \times 3,05 \mathrm{~m} \\
\mathrm{Fc}-0,56 \\
\mathrm{Kc}-1,10 \\
\mathrm{UE}-0,90 \\
\mathrm{TR}-5 \text { dias }\end{array}$} & 3,70 & 20,53 & 8,62 \\
\hline 3 anos & $70 \%$ & 7,25 & & 3,82 & 21,22 & 8,91 \\
\hline 5 anos & $80 \%$ & 7,37 & & 3,88 & 21,56 & 9,06 \\
\hline 10 anos & $90 \%$ & 7,44 & & 3,94 & 21,90 & 9,20 \\
\hline 20 anos & $95 \%$ & 7,64 & & 4,07 & 22,59 & 9,49 \\
\hline \multicolumn{2}{|c|}{ Média mensal de setembro } & 5,33 & & 3,26 & 18,14 & 7,62 \\
\hline \multirow{3}{*}{ Período de retorno } & \multirow{3}{*}{$\begin{array}{l}\text { Probabilidade } \\
\text { de ocorrência }\end{array}$} & \multirow{3}{*}{$\begin{array}{c}\mathrm{ETO}_{1} \\
\mathrm{~mm}\end{array}$} & \multirow{2}{*}{$\begin{array}{c}\text { Parâmetros do projeto } \\
\text { de irrigação }\end{array}$} & ETc & ITN & VTN \\
\hline & & & & $\mathrm{mm}$ & $\mathrm{mm} \mathrm{dia}{ }^{-1}$ & L planta ${ }^{-1}$ dia $^{-1}$ \\
\hline & & & \multicolumn{4}{|c|}{ Tomate - irrigação por gotejamento com faixa molhada contínua } \\
\hline 2 anos & $50 \%$ & 8,18 & \multirow{6}{*}{ 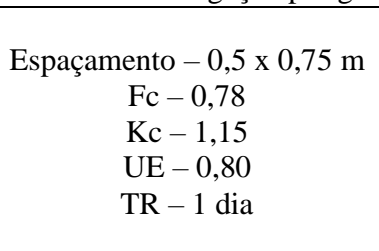 } & 5,35 & 6,68 & 2,51 \\
\hline 3 anos & $70 \%$ & 8,30 & & 5,53 & 6,91 & 2,59 \\
\hline 5 anos & $80 \%$ & 8,38 & & 5,61 & 7,02 & 2,63 \\
\hline 10 anos & $90 \%$ & 8,43 & & 5,70 & 7,13 & 2,67 \\
\hline 20 anos & $95 \%$ & 8,57 & & 5,88 & 7,35 & 2,76 \\
\hline \multicolumn{2}{|c|}{ Média mensal de setembro } & 5,33 & & 4,72 & 5,90 & 2,21 \\
\hline
\end{tabular}

Fc - Porcentagem de área coberta pela cultura; Kc - Coeficiente da cultura máximo; UE - uniformidade de emissão; TR - Intervalo entre irrigações; ETo5 - Evapotranspiração média acumulada de 5 dias; ETo1 - Evapotranspiração média acumulada de 1 dia; ETc Evapotranspiração da cultura, considerando o Fc; ITN - Irrigação diária total necessária; VTN - Volume bruto de irrigação por planta por dia. *Adaptado de: Frizzone et al. (2012)

Caso se deseje utilizar a probabilidade de $90 \%$, uma vez que, normalmente, níveis elevados somente são adotados para culturas irrigadas de grande valor econômico e com sistema radicular pouco profundo (JENSEN, 1989), ainda sim, haveria o subdimensionamento em cerca de $29 \%$ e $37 \%$ por parte do valor médio para a cultura do café e tomate, respectivamente.

\section{CONCLUSÕES}

As distribuições de evapotranspiração de referência acumulada ajustaram-se aos modelos de distribuições Log-Normal, Weibull, Gama, Normal e Logística. O valor de evapotranspiração de referência acumulada indicado para dimensionar os sistemas de irrigação na Bacia Hidrográfica do Alto Juruá, foi de 7,37, 8,38 e $6,34 \mathrm{~mm} \mathrm{dia}{ }^{-1}$, correspondendo ao período acumulado de 1, 5 e 20 dias, respectivamente.

\section{REFERÊNCIAS}

ANA - Agência Nacional de Águas. Atlas irrigação: Uso da água na agricultura irrigada. Brasília, ANA, 2017. $86 \mathrm{p}$.

ALBUQUERQUE, P.E.P.; DURÃES, F.O.M. Uso de manejo de irrigação. Embrapa Informação Tecnológica, Brasília. 2008. 528 p.

ALLEN, R.G.; PEREIRA, L.S.; RAES, D.; SMITH, M. Crop evapotranspiration: Guidelines for computing crop water 
requirements, FAO. Irrigation and Drainage Paper - 56. FAO, Rome. 1998.

ALVARES C.A.; STAPE, J.L.; SENTELHAS, P.C.; GONÇALVES, J.L M.; SPAROVEK, G. Köppen's climate classification map for Brazil. Meteorologische Zeitschrift, v. 22, n. 6, p. 711-728, 2013.

AUGUIE, B. gridExtra: Miscellaneous Functions for "Grid" Graphics. R package version 2.3. 2017.

BACK, A.J. Variação da evapotranspiração de referência calculada em diferentes intervalos de tempo. Engenharia Agrícola, v. 27, n. 1, p.139-145. 2007.

BELlOSTA, C.J.G. ADGofTest: AndersonDarling GoF test. $\mathrm{R}$ package version 0.3. 2011.

BERNARDO, S.; SOARES, A. A.; MANTOVANI, E. C. Manual de Irrigação. 8. Ed. Viçosa. Viçosa. 2009. 625 p.

CÓRCOLES, J.I.; JUAN, J.A.; ORTEGA, J.F.; TARJUELO, J.M.; MORENO, M.A. Management evaluation of Water Users Associations using benchmarking techniques. Agricultural Water Management, v. 98, n. 1, p. 1-11, 2010.

DELIGNETTE-MULLER,

M.L.; DUTANG,C. fitdistrplus: An R Package for Fitting Distributions. Journal of Statistical Software, v. 64, n. 4, p.1-34, 2015.

DENSKI, A.P.N.; BACK, A.J. Aderência de distribuições de probabilidade de evapotranspiração de referência decendial. Revista Brasileira de Climatologia, v. 17, n. 11, p. 26-40. 2015.

FERNANDES, R.D.M.; JOSÉ, J.V.; WOLFF, W.; COSTA, J.O.; FOLEGATTI, M.V.; Probability distribution functions applied in the water requirement estimates in irrigation projects. Revista Caatinga, v. 32, n. 1, p. 189199, 2019.

FIETZ, C.R.; FRIZZONE, J.A.; PINTO, J.M. Probabilidade de ocorrência da evapotranspiração na região de Dourados, MS. Ciência Rural, Santa Maria, v. 27, n. 2, p. 207-210, 1997.

FRIZZONE， J.A.; FREITAS， P.S.L.; REZENDE, R.; FARIA, M.A. Microirrigação: gotejamento e microaspersão. Eduem. Maringá. 2012. 356 p.

HIJMANS, R.J. raster: Geographic Data Analysis and Modeling. R package version 3.1-5. 2020.

IBGE - Instituto Brasileiro de Geografia e Estatística. Censo agropecuário - 1995-1996. Rio de Janeiro, 1998.

IBGE - Instituto Brasileiro de Geografia e Estatística. Manual do Recenseador CA - 1.09: Censo Agropecuário - 2017. Rio de Janeiro: IBGE. 2017. 147p.

JENSEN, M.E.; BURMAN, R.D.; ALLEN, R.G. Evapotranspiration and irrigation water requirements. ASCE. New York. 1989. $332 \mathrm{p}$.

JOSÉ, V.J.; SANTOS, L.C.; ALVES, D.S.; NITSCHE, P.R.; FOLEGATTI, M.V.; WOLFF, W. Aspectos espaciais da evapotranspiração com o foco no dimensionamento de sistemas de irrigação. Irriga, v. 24, n. 4, p. 781-801, 2019.

NAGHETTINI, M.; PINTO, E.J.A. Hidrologia estatística. Belo Horizonte: CPRM. 2007. 552p.

PIERCE, D. ncdf4: Interface to Unidata netCDF (Version 4 or Earlier) Format Data Files. R package version 1.17. 2019.

PRUITT, W.O.; OETTINGEN， S.V.; 
MORGAN, D.L. Central California: Evapotranspiration frequencies. Journal of the Irrigation and Drainage Division, v. 98, n. IR-2, p.177-184, 1972.

$\mathrm{R}$ CORE TEAM. R: A language and environment for statistical computing. Vienna, Austria: R Foundation for Statistical Computing. 2020.

SAAD, J.C.C.; BISCARO, G.A.; DELMANTO, O.J.; FRIZZONE, J. A. Estudo da distribuição da evapotranspiração de referência visando o dimensionamento de sistemas de irrigação. Irriga, v. 07, n. 1, p. 1017, 2002.

SAAD, J.C.C.; SCALOPPI, E.J. Freqüência de distribuição de evapotranspiração para dimensionamento de sistemas de irrigação. In: Congresso Nacional de Irrigação e Drenagem, 8, 1988. Florianópolis, SC. Anais... Florianópolis: ABID, v. 2, p. 1037-1052.

SANTOS, N.T.; SILVA, M.G.; OLIVEIRA, A.S.; SILVA, J.A.; PEREIRA, F.A.; BATISTA, L.S. Distribuição de probabilidade da evapotranspiração de referência em Cruz das Almas, Bahia. Anais... II Inovagri International Meeting, Fortaleza, 2014.

SANTOS, W.O.; BATISTA, B.D.O.; SOBRINHO, J.E.; PEREIRA, V.C.; JUNIOR, E.C.; ALMEIDA, B.M. Ajuste de modelos de distribuição de probabilidade a série histórica de 1970 a 2007 de evapotranspiração de referência (ETo) em Mossoró-RN.In: Congresso Brasileiro de Agrometeorologia, XVII, Guarapari, 2011.

SILVA, M.G.; BATISTA, L.S.; CARMO, F.F.; SANTOS, N.T.; OLIVEIRA, I.S. Probabilidade de ocorrência de valores estimados de evapotranspiração de referência no sertão do Ceará. Revista Engenharia na Agricultura, v. 22, n. 6, p. 562-574, 2014.

WICKHAM, H. ggplot2: Elegant Graphics for Data Analysis. Springer-Verlag New York, 2016.

XAVIER, A.C.; KING, C.W.; SCANLON, B.R. Daily gridded meteorological variables in Brazil (1980-2013). International Journal of Climatology, v. 36, n. 6, p. 26442659, 2015. 